\title{
Mental health of adolescents with type I diabetes: A case report
}

\author{
Efterpi Marini, George Giannakopoulos, Stella Charitaki, Maria Belivanaki, Varvara Salavou, \\ Magda Liakopoulou, Gerasimos Kolaitis
}

Department of Child Psychiatry, Athens University Medical School, “Aghia Sophia” Children’s Hospital, Athens, Greece;

*Corresponding Author: gkolaitis@med.uoa.gr

Received 16 January 2013; revised 19 February 2013; accepted 19 March 2013

Copyright (c) 2013 Efterpi Marini et al. This is an open access article distributed under the Creative Commons Attribution License, which permits unrestricted use, distribution, and reproduction in any medium, provided the original work is properly cited.

\section{ABSTRACT}

Type I Diabetes Mellitus (DM I) is the third most common chronic childhood disease and can cause both short-term and long-term complications, as well as acute life-threatening events. The announcement of the DM I diagnosis in childhood or adolescence constitutes a major psychosocial stressor for the child and his family. Diabetes in general complicates the parentchild relationship and increases adolescents' negative thoughts about themselves and depressive mood. The majority of patients experience depressive and/or anxiety symptoms at the time of diagnosis, which generally resolve within six to nine months. Poor adjustment in this initial phase places adolescents at risk for later psychosocial difficulties. On a long-term basis, individuals with DM I may exhibit significant psychiatric and behavioral problems including depression, anxiety and anger. We here reported the case of a 13-year-old boy, suffering from DM I for 3 years, who developed poor metabolic control, depressive symptoms and life-threatening behavior in the past 6 months and was hospitalized at an inpatient psychiatric unit. The complex biopsychosocial needs of adolescents with Type I Diabetes Mellitus should be recognized and addressed through tailored interventions by medical and mental health professionals.

Keywords: Diabetes Mellitus Type 1; Adolescence; Depression

\section{INTRODUCTION}

Type I Diabetes Mellitus (DM I), the incidence of which continues to increase worldwide $[1,2]$, is the third most common chronic disease in childhood [3]. It is an autoimmune disease, characterized by destruction of the insulin-producing beta cells in the pancreas, leading to total or near total insulin deficiency. The disease has a bimodal presentation regarding age of onset, with the first peak between 4 and 6 years of age and the second peak in early adolescence [1,4,5]. Diabetes can cause both short-term and long-term complications, as well as acute life-threatening events (hypoglycemia, diabetic ketoacidosis) [4-6]. The treatment for DM I includes close monitoring of blood glucose levels and multiple insulin injections per day, in conjunction with a healthy lifestyle $[1,4]$. Intensive management is known to reduce diabetic complications and to improve long-term health outcomes [7]. During adolescence, deterioration in metabolic control, often attributable to erratic meal and exercise patterns, poor adherence to treatment regimens, hazardous and risk taking behaviors, as well as endocrine changes associated with puberty (growth hormone and insulinlike growth factor-1) lead to great insulin resistance and poor response to insulin treatment $[3,5,8]$.

The announcement of the DM I diagnosis in childhood or adolescence constitutes a major psychosocial stressor for the child and their family, as both are introduced to a new world filled with challenges, constraints and uncertainties associated with a lifelong illness [5,9,10]. Diabetes, in general, complicates the parent-child relationship and increases the adolescent's negative thoughts and depressive mood [5,11,12].

The prevalence of depression or depressive symptoms in diabetic children and adolescents ranges from 2 to 3 times that of their non-diabetic peers [13-15]. When DM I and depression coexist, poor compliance with diabetes care is frequent, resulting in poor glycaemic control and increased risk of long-term complications [16].

We presented the case of A., a 13-year-old boy, suffering from DM I, who developed poor metabolic control, 
depressive symptoms and life-threatening behavior in the past 6 months. The admission to an inpatient psychiatric unit at a Children's Hospital was considered essential at that time. We here described the course of his illness during hospitalization, the serious affective manifestations interfering with his daily diabetic care and the way he achieved better metabolic control once his psychiatric condition improved.

\section{CASE REPORT}

A. has suffered from DM I since the age of 11 years. During the last year his behavior had changed, and his mental state was significantly impaired, especially in the past six months. He displayed severe agitation and irritation, social withdrawal and academic impairment. A. became careless of diabetic control, did not follow medical instructions on insulin treatment, showed no regimen adherence, smoked tobacco and consumed alcohol almost daily. The result of this behavior was evident in the glycated hemoglobin (HbA1c) level, which was 13\% prior to his hospitalization. He was isolated, feeling alone among peers and had no close friends. In the last six weeks, he abandoned all his hobbies and experienced severe conflicts with his siblings and parents. He would stay alone in his bedroom for hours and refuse to take part in the family meals or would spend many hours outside the home. A. was totally detached from his siblings in the last months and resented his parents for prioritizing his younger siblings' satisfaction. He suffered from the feeling that he is the only one in his family with problems, as none of his siblings had any physical illness. At times of intense anger he would harm himself with sharp tools, wishing to die, while his parents felt hopeless and unable to cope with this difficult situation.

A. was first admitted to the pediatric ward for full physical examination. The assessment by child psychiatrists led to a diagnosis of Major Depressive Disorder and it was considered that there was a high risk for harming himself, which urged his admission to the in-patient psychiatric unit.

\subsection{Developmental and Family History}

A. is the third child of a 6-member family. At 9-dayold, he presented with a urinary tract infection and was admitted to a neonatal intensive care unit. An ultrasound revealed a congenital solitary missing kidney. Because A. suffered from cow's milk allergy, his mother followed an especially strict diet in order to breastfeed him. At the age of 9 , hypothyroidism was diagnosed and $\mathrm{T}_{4}$ treatment was initiated. Two years later, he presented with polydipsia and polyuria and DM I was diagnosed.

Although his kindergarten teacher had noticed some inappropriate bursts of anger, his adjustment was de- scribed as normal. A. attended the last three years of elementary school abroad, as their family moved to another country due to father's profession. No problems in his adjustment to the new school environment and peers were reported. Upon his entrance in high school, his family repatriated. Even though the return to his homeland was not easy for A., he managed to perform well at school, study foreign languages, play the guitar and train in sports. Nevertheless, A. faced difficulties in finding new friends.

The father presented a Major Depressive Episode at the age of 20 and two close relatives had suffered from mood disorders. Both parents had also received multiple psychotherapy interventions in the past due to depressive and anxiety symptoms as well as marital difficulties. The father seemed to understand A's emotional needs and to be close to him. His mother, on the other hand, showed low emotional involvement and used psychological interpretations for every single event in everyday life. She had not adequately adjusted to her son's chronic illness and denied his difficulties.

\subsection{Psychiatric Evaluation}

Upon admission to the inpatient psychiatric unit, A. presented as a typical rebellious teenager, with general appearance and clothing being appropriate to his age, characterized by intense depressive affect. He was provocative towards the mental health staff, avoiding eye contact and being reluctant to answer their questions. He was oppositional and agitated, feeling distressed and indifferent. No disorders of thought, speech and perception were detected. No disturbances in sleep or food appetite were reported. A. tried to omit insulin injections or, when he was obliged to cooperate, he used to inject the medicine over his clothes. He was impolite and offensive with the staff and wary of other hospitalized children. He also avoided any contact with his parents.

Intelligence quotient was within the normal range. Clinical examination and the psychiatric semi-structured interview K-SADS-PL $[17,18]$ were performed. According to the Diagnostic and Statistical Manual of Mental Disorders DSM-IV-TR [19], the diagnosis of double depression (Major Depression and Dysthymic Disorder) was made.

\subsection{Management and Treatment}

During his hospitalization, A. received medical and psychosocial treatment. Since antidepressant agents provoked insulin resistance, the relative medication was initiated under close endocrinology monitoring, and only after metabolic control was achieved. We initially administered Fluvoxamine at $50 \mathrm{mg}$ daily which was then increased to $100 \mathrm{mg}$ per day. A. attended individual 
psychotherapy sessions in addition to the pharmacological treatment. His parents and the family received counselling sessions. Progressively, he came to recognize the need for his hospitalization and to trust the therapeutic milieu. A. started to comply with the treatment, the diabetes hygiene and the special regimen. Self-harming ideas were reduced, his mood was improved, and this was also the case for his metabolic control (tested by HbA1c: 6.7\%). His interactions with his family and peers improved, he became friendly and spontaneous and took on a leadership role in group activities.

During A.'s hospitalization, parents worked through their perceptions of the chronic illness and their understanding of their offspring's emotional and somatic needs increased. His parents eventually managed to associate A's maladjustment issues with their own difficulty in accepting his chronic disease. Upon discharge, the continuation of individual and family therapy in addition to pharmacological treatment were recommended.

\section{DISCUSSION}

Diabetes is a complex disease to manage, with physical, economic and mental health effects, requiring knowledge about the disease, intense treatment and skills in everyday tasks (insulin injection, glucose testing) in order to avoid long-term and life threatening complications [5,6]. A developmentally appropriate understanding of the nature of diabetes is essential. A child's refusal to comply with treatment may represent misunderstanding, fear and concern, which, if initially detected by the clinician, may provide an opportunity to explore the reasons for non compliance and to strength the therapeutic relationship [5]. During adolescence, children come to realize the chronicity of the illness and become anxious and/ or depressed. This is especially true for adolescents for whom the challenge of diabetes is combined with the developmental task of adapting to puberty and changing body image, peer group pressure, autonomy from parents and identity formation [14]. Although adolescents strive to show more independence, they may not be ready for complete independence in their diabetic care [15].

Depression is a serious health problem that may affect individuals suffering from chronic illnesses. The majority of patients experience depressive symptoms and/or anxiety at the time of diagnosis of DM I, which generally resolve within six to nine months [5]. Poor adjustment in this initial phase places children at risk for later psychosocial difficulties [14]. On a long-term basis, individuals with DM I exhibit significant psychiatric and behavioral issues including depression, anxiety and anger. The prevalence of depressive symptoms in young people with diabetes is $12 \%$ in children aged 8 - 12 years and $18 \%$ in adolescents $[14,20]$. Loss of health, complex treatment recommendations, fear of hypo-/hyperglycemia and interference with family lifestyle are sources of diabetesrelated stress that may contribute to the development of depression over time [21]. Depression may result in behavioral or personality changes such as low self esteem, pessimism, poor concentration, and loss of interest in daily activities [14]. Various studies suggest that the comorbitity of depression and DM I in adolescents may be due to hormonal changes, insulin resistance of puberty, eating disorders, the possibility of insulin delivery errors, experimentation with alcohol and tobacco, as our patient does, and, last but not least, continuous demand for adjustment and adaptation to school and leisure. Studies in animals with DM I found progressive atrophy of hippocampal function similar to that found in depressive illness [15]. Diabetes is also associated with white matter lesions thought to increase risk for "vascular depression" [7]. Other studies associate maternal depression with higher incidence of depression in children with diabetes [14,21].

Parents of children with DM I are in face with several potential sources of stress. Upon the diagnosis, they must come to terms with the loss of health for their previously healthy child. They have to deal with the DM I and its implications as well as the likelihood of shortened life expectancy [21]. The relationship between family life and diabetic control is also well studied. It has been shown that it is an interactive one, with each affecting and in turn being affected by other. Patients and their parents should be aware from the beginning that the prevalence of depression in patients with diabetes is higher than in the general population. Involving parents in the care of the child or adolescent with diabetes is beneficial to improving metabolic control because improvements in family communication and problemsolving skills decrease stress in the diabetic adolescent. The difficult task is to determine exactly how much involvement a parent should have in the adolescent's care [15]. Protective factors such as family communication skills, spousal support and enhancement of positive selfperception should be identified and promoted to minimize short- and long-term complications [5].

The psychosocial adjustment and behavior of patients with DM I are critical to their medical outcomes and quality of life. Interventions in adolescents with DM I, including individual psychotherapy, educational programs, cognitive behavior therapy, coping-skills training and family based interventions showed promise in reducing family conflict and achieving metabolic control. Peer group intervention is beneficial as peers are more likely to provide emotional support and companionship in relation to the care of diabetes than family members [15]. There is no current contraindication to using drug therapy in treating depression in diabetic patients with a slow 
dosage adjustment.

As adolescents with DM I are characterized by frequent alteration between dependence and independence conflicts from the family, agony about their ability to cope with the disease and fear of death, psychosocial complications' identification and intervention may prevent future relapse in adolescents.

\section{REFERENCES}

[1] Haller, M.J., Atkinson, M.A. and Schatz, D. (2005) Type 1 diabetes mellitus: Etiology, presentation, and management. Pediatric Clinics of North America, 52, 1553-1578. doi:10.1016/j.pcl.2005.07.006

[2] Karvonen, M., et al. (2000) Incidence of childhood type 1 diabetes worldwide. Diabetes Mondiale (DiaMond) Project Group. Diabetes Care, 23, 1516-1526. doi:10.2337/diacare.23.10.1516

[3] McNamara, R., et al. Development and evaluation of a psychosocial intervention for children and teenagers experiencing diabetes (DEPICTED): A protocol for a cluster randomised controlled trial of the effectiveness of a communication skills training programme for healthcare professionals working with young people with type 1 diabetes,” BMC Health Services Research, 10, 36. doi:10.1186/1472-6963-10-36

[4] Tamborlane, W.V. and Ahern, J. (1997) Implications and results of the diabetes control and complications trial. $\mathrm{Pe}$ diatric Clinics of North America, 44, 285-300. doi:10.1016/S0031-3955(05)70477-6

[5] Fritsch, S.L., Overton, M.W. and Robbins, D.R. (2011) The interface of child mental health and juvenile diabetes mellitus. Pediatric Clinics of North America, 58, 937-954. doi:10.1016/j.pcl.2011.06.008

[6] Desrocher, M. and Rovet, J. (2004) Neurocognitive correlates of type 1 diabetes mellitus in childhood. Child Neuropsychology, 10, 36-52. doi:10.1076/chin.10.1.36.26241

[7] Levenson, J.M. and Sweatt, J.D. (2006) Epigenetic mechanisms: A common theme in vertebrate and invertebrate memory formation. Cellular and Molecular Life Sciences, 63, 1009-1016. doi:10.1007/s00018-006-6026-6

[8] Drotar, D. and Ievers, C. (1994) Age differences in parent and child responsibilities for management of cystic fibrosis and insulin-dependent diabetes mellitus. Journal of Developmental \& Behavioral Pediatrics, 15, 265-272. doi:10.1097/00004703-199408000-00008

[9] Northam, E.A., Todd, S. and Cameron, F.J. (2006) Interventions to promote optimal health outcomes in children with Type 1 diabetes-Are they effective? Diabetic Medicine, 23, 113-121.

\section{doi:10.1111/j.1464-5491.2005.01678.x}

[10] Northam, E., et al. (1996) Psychosocial and family functioning in children with insulin-dependent diabetes at diagnosis and one year later. Journal of Pediatric Psychology, 21, 699-717. doi:10.1093/jpepsy/21.5.699

[11] Kovacs, M., et al. (1985) Initial coping responses and psychosocial characteristics of children with insulin-dependent diabetes mellitus. Journal of Pediatrics, 106, $827-$ 834. doi:10.1016/S0022-3476(85)80368-1

[12] Liss, D.S., et al. (1998) Psychiatric illness and family support in children and adolescents with diabetic ketoacidosis: A controlled study. Journal of the American Academy of Child \& Adolescent Psychiatry, 37, 536-544. doi:10.1097/00004583-199805000-00016

[13] Levenson, J.L. (2006) Psychiatric issues in endocrinology: Updates in psychosomatic medicine and consultation-liaison psychiatry. Primary Psychiatry, 13, 27-30.

[14] Whittemore, R., et al. (2002) Correlates of depressive symptoms in adolescents with type 1 diabetes. Pediatric Diabetes, 3, 135-143. doi:10.1034/j.1399-5448.2002.30303.x

[15] Massengale, J. (2005) Depression and the adolescent with type 1 diabetes: The covert comorbidity. Issues in Mental Health Nursing, 26, 137-148. doi:10.1080/01612840590901590

[16] Kamboj, M.K. and Tareen, R.S. (2011) Management of nonpsychiatric medical conditions presenting with psychiatric manifestations. Pediatric Clinics of North America, 58, 219-241. doi:10.1016/j.pcl.2010.10.008

[17] Kolaitis, G., et al. (2003) Schedule for affective disorders and schizophrenia for school-age children-present episode (K-SADS-P): A pilot inter-rater reliability study for Greek children and adolescents. European Psychiatry, 18, 374-375. doi:10.1016/j.eurpsy.2003.02.002

[18] Kaufman, J., et al. (2000) K-SADS-PL. Journal of the American Academy of Child \& Adolescent Psychiatry, 39, 1208. doi:10.1097/00004583-200010000-00002

[19] APA (2000) Diagnostic and statistical manual of mental disorder. 4th Edition, American Psychiatric Association, Washington DC.

[20] Kokkonen, K. (1995) Mental health and social adaptation in young adults with juvenile-onset diabetes. Nordic Journal of Psychiatry, 49, 175-181. doi:10.3109/08039489509011903

[21] Kovacs, M., et al. (1990) Psychological functioning among mothers of children with insulin-dependent diabetes mellitus: A longitudinal study. Journal of Consulting and Clinical Psychology, 58, 189-195. doi:10.1037/0022-006X.58.2.189 